\title{
La Fundación IAVANTE, y su apuesta por el e.Learning
}

\author{
Carmen Blanco*, Guillermo Vázquez ** \\ *Directora Gerente de la Fundación IAVANTE **Director de I+D de la Fundación IAVANTE
}

El II Plan de Calidad del Sistema Sanitario Público de Andalucía $^{a}$ 2005-2008, contempla entre sus procesos estratégicos la "GESTIÓN DEL CONOCIMIENTO”. Esto se traduce en la necesidad de identificar el mejor conocimiento médico y enfermero así como las habilidades que se requieren para aplicarlo en su práctica diaria, y transferirlo no solo a los profesionales de la sanidad, sino también a los ciudadanos. Paralelamente atribuye a las Tecnologías de la Información y la Comunicación (TICs) una función central en la innovación de nuestra sanidad en todas sus vertientes. Los conceptos de e.Salud, e.Ciudadano y e.Formación (e.Learning) se convierten en objetivos de centrales de la Sociedad de la Información, y descansan sobre las TICs.

La Fundación IAVANTE, creada y dependiente por la Consejería de Salud de Andalucía en el año 2002, se engarza en ambos contextos descritos, al tener como misión central identificar, transferir, y compartir el conocimiento clínico, sus habilidades inherentes, y considerar la TICs una de sus herramientas fundamentales. La actividad de la Fundacion se centra predominantemente en la vertiente asistencial, del Sistema Público de Salud de Andalucía. Para cumplir su misión la Fundación IAVANTE se apoya en dos pilares el primero es el entrenamiento de los profesionales recurriendo a las metodologías de aprendizaje más actualizadas, entre ellas la robótica y el e.Learning; el segundo eje es su promoción e implantación. El conocimiento para promover estas acciones, lo adquiere de los profesionales del Servicio Público Andaluz y de las empresas de nuestros Parques Tecnológicos, mediante alianzas y cooperación. Actualmente la Fundacion IAVANTE, como consecuencia de su apuesta por la "formacion online" posee un Campus Virtual accesible desde cualquier centro hospitalario o centro de medicina familiar de Andalucía. Este Campus Virtual ofrece un amplio repositorio de acciones formativas online o presenciales para los profesionales interesados en mejorar su formacion en competencias; paralelamente también ofrece con un sistema de apoyo al diseño de acciones formativas y gestión de los recursos necesarios para impartirlas, dirigido a los profesores $\mathrm{y}$ tutores de la Fundacion. Esta plataforma operativa de software libre denominada SIGESXCOM, se complementa con varias Web-sites específicas para determinados grupos de sanitarios, como por ejemplo residentes de nuestro Sistema Sanitario Público de Andalucía. Otras acciones en marcha en nuestro Campus Virtual es la creación de comunidades virtuales mediante instrumentos colaborativos como son los WIKIs, sirviendo como ejemplo el apoyo al Consejo Asesor Científico de la Fundacion IAVANTE, formado por las Sociedades Científicas Sanitarias de Andalucía, denominado WIKI-CACIFI.

Anualmente la Fundacion IAVANTE promueve reuniones científicas del área educativa en las que la participación de especialistas de prestigio internacional, le permite identificar las tendencias e innovaciones mas actualizadas y eficientes en pedagogía, metodología y tecnología educativas. Los contenidos de esta monografía corresponden a los temas expuestos en el II Symposium Internacional IAVANTE 2005, desarrollado en el Centro Multifuncional Avanzado de Simulación e Innovación Tecnológica que la Fundacion IAVANTE posee en el Parque de ciencias de Salud de Granada. Este Symposium examinó en profundidad el e.Learning y secundariamente la telemedicina, dada la íntima conexión entre ambas. El tema este Symposium Internacional IAVANTE, conecta con los objetivos del II Plan de Salud de Andalucía, y con los objetivos de la Unión Europea en relación a la potenciación de las TICs y su aplicación en el campo de la Sanidad.

Esta monografía complementa perfectamente los objetivos de nuestro Simposium, y la misión de nuestra Fundacion, al contribuir a diseminar el mejor conocimiento científico educativo entre los profesionales de la Sanidad. Las TICs, aplicadas a la educación, formación y entrenamiento de los profesionales médicos y enfermeros, supondrá una innovación con repercusión en muchas áreas de la salud y enfermedad; su alcance y trascendencia se esta visualizando en la Fundacion IAVANTE por el incremento exponencial de profesionales sanitarios que encuentran en su Campus Virtual, una herramienta cómoda y adecuada a sus necesidades de formación y entrenamiento. 